\title{
Schwinger Functions for the Yukawa Model in Two Dimensions with Space-Time Cutoff*
}

\author{
Erhard Seiler $\star \star$ \\ Institute for Advanced Study, Princeton, New Jersey, USA \\ Received July 15, 1974; in revised form January 20, 1975
}

\begin{abstract}
It is shown that a Euclidean version of the formulae of Matthews and Salam for the Green's functions of a two-dimensional Yukawa model with interaction in a finite space-time volume makes sense, if renormalized correctly.
\end{abstract}

\section{Introduction}

Twenty years ago Matthews and Salam [1] presented formulae for the Green's functions of a Yukawa theory which arose from formally integrating out the fermions in Feynman's "sum over histories" [2]. Those formulae expressed the Green's functions as functional integrals over the Bose field of certain combinations of determinants.

In this paper we show that a Euclidean version of those formulae for a twodimensional Yukawa (" $Y_{2}$ ") model with a space-time cutoff for the interaction actually makes sense, if the necessary renormalization is carried out in the appropriate way.

We do not discuss here the problem of removing the space-time cutoff which would hopefully lead to Schwinger functions fulfilling all the axioms of Osterwalder and Schrader $[3,4]$ guaranteeing the existence of the corresponding quantum field theory fulfilling Wightman's axioms.

Recently Osterwalder and Schrader [5] have constructed Euclidean Fermi fields and proved a Feynman-Kac type formula which relates Euclidean expressions in a finite space-time valume (and containing ultraviolet cutoffs) to quantities of the "physical" $Y_{2}$ theory. This gives a connection between our integrability statements and the semiboundedness of the $Y_{2}$ Hamiltonian which was first proved by Glimm [6] (see also Glimm and Jaffe [7], Schrader [8], Brydges and Federbush [9], and Brydges [10]). But we are not able to construct an independent proof of this semi-boundedness at this stage; this would involve the removal of at least the time cutoff.

Osterwalder's and Schrader's fields can also be used to derive an ultraviolet and space-time cutoff version of the Matthews-Salam formula (1.1) which we take here simply as a heuristic starting point. This derivation also guarantees the physical ("Osterwalder-Schrader") positivity of the approximate Schwinger functions (see [5]) for suitably chosen space-time cutoff.

* This work was supported in part by NSF Grant No. GP-16147 A No. 1.

$\star \star$ On leave of absence from Max-Planck-Institut für Physik und Astrophysik, München, Federal Republic of Germany. 
Our starting point is the following expression for the Schwinger functions with a space-time cutoff (compare [1]):

$$
\begin{aligned}
S_{g}\left(x_{1}, \ldots, x_{n} ; y_{1}, \ldots y_{m} ;\right. & \left.z_{1}, \ldots, z_{m}\right) \\
= & \frac{1}{Z} \int_{\mathscr{S}^{\prime}} d \mu_{0}(\Phi) \operatorname{det}_{i k} S_{F}{ }^{\prime}\left(y_{i}, z_{k} ; \phi_{g}\right) \prod_{l=1}^{n} \Phi\left(x_{l}\right) \\
& \cdot \operatorname{det}_{\mathrm{ren}}\left(1+\lambda K\left(\Phi_{g}\right)\right),
\end{aligned}
$$

where

$$
Z=\int d \mu_{0}(\Phi) \operatorname{det}_{\text {ren }}\left(1+\lambda K\left(\Phi_{g}\right)\right)
$$

$d \mu_{0}(\Phi)$ denotes the functional measure for the free Euclidean Bose field of mass $\mu$ (see e.g. Nelson [11]; Guerra, Rosen, and Simon [12]) which can be realized on $\mathscr{S}^{\prime}$, the space of tempered distributions. $S_{F}{ }^{\prime}\left(x, y ; \Phi_{g}\right)$ denotes the two point Schwinger function for the fermions in the external field $\Phi_{g}(x)=\Phi(x) g(x)$, where $g(x)$ is a suitable spacetime cutoff function (e.g. $g \in C_{0}^{\infty}$, or $g=\chi_{A}$, the characteristic function of a bounded region $\left.\Lambda \subset \mathbb{R}^{2}\right) . K\left(\Phi_{g}\right)$ is the "integral" operator occuring in the "integral" equation for $S_{F}{ }^{\prime}\left(x, y ; \Phi_{g}\right)$ :

where

$$
(1+\lambda K) S_{F}{ }^{\prime}=S_{F}
$$

$$
S_{F}=\frac{1}{(2 \pi)^{2}} \int \frac{\not p+m}{p^{2}+m^{2}} e^{i p x} d^{2} p
$$

is the two point Schwinger function for the fermions. $K\left(\Phi_{g}\right)$ has the kernel

$$
K\left(\Phi_{g}\right)(x, y)=S_{F}(x-y) \Gamma \Phi(y) g(y) \quad\left(\Gamma=1 \quad \text { or } \quad i \gamma_{5}\right)
$$

(since $S_{F}$ and $\Phi$ are distributions, $K$ is not really an integral operator; see Section 2). $\operatorname{det}_{\text {ren }}(1+\lambda K)$ denotes a suitably renormalized Fredholm determinant which will be defined in the next section. Our conventions for the $\gamma$-matrices are the following:

and, of course

$$
\begin{aligned}
\gamma_{\mu}^{*} & =-\gamma_{\mu} \quad(\mu=0,1) \\
\left\{\gamma_{\mu}, \gamma_{\nu}\right\} & =-2 \delta_{\mu \nu} \\
\gamma_{5} & =i \gamma_{0} \gamma_{1}=\gamma_{5}^{*}
\end{aligned}
$$

$$
\not p=p_{0} \gamma_{0}+p_{1} \gamma_{1}
$$

\section{Definition of the Renormalized Fredholm Determinant}

First we want to show that the kernel $K\left(\Phi_{g}\right)(1.5)$ defines almost everywhere (with respect to $d \mu_{0}$ ) a compact operator on the Hilbert space

where

$$
\mathscr{H}=\mathscr{H}_{\frac{1}{2}} \oplus \mathscr{H}_{\frac{1}{2}}=\mathbb{C}^{2} \otimes \mathscr{H}_{\frac{1}{2}},
$$

$$
\mathscr{H}_{\frac{1}{2}}=L^{2}\left(\sqrt{p^{2}+m^{2}} d^{2} p\right) \text {. }
$$

This will follow from a general fact about operator valued random variables and the finiteness of certain Feynman graphs. Let $\mathscr{C}_{p}$ denote the set of compact 
operators $C$ on $\mathscr{H}$ with $\|C\|_{p}^{p}:=\operatorname{Tr}\left(C^{*} C\right)^{p / 2}<\infty$. We now consider operator valued functions

$$
A: \mathscr{S}^{\prime} \rightarrow \mathscr{C}_{p}
$$

which are weakly measurable ${ }^{1}$ and we define

$$
\|A\|_{p ; q}=\left(\int d \mu_{0}\|A(\Phi)\|_{p}^{q}\right)^{1 / q} .
$$

Lemma 2.1. The spaces $\mathscr{C}_{p ; q}=\left\{A: \mathscr{S}^{\prime} \rightarrow \mathscr{C}_{p} \mid A\right.$ weakly measurable, $\left.\|A\|_{p ; q}<\infty\right\}$ $(p, q \geqq 1)$ are complete.

Proof. This can be shown in exactly the same way as the completeness of the ordinary $L_{p}$-spaces, using the fact that $\mathscr{C}_{p}$ is complete (see Schatten [13]).

We now introduce a sequence of ultraviolet cutoff functions $h_{n} \in C^{\infty}$ for the bosons:

$$
\begin{gathered}
h_{n}(x)=\frac{1}{(2 \pi)^{2}} \int e^{i p x} \hat{h}_{n}(p) d^{2} p \quad(n=1,2,3, \ldots) \\
\hat{h}_{n}(p) \in C_{0}^{\infty} ; \hat{h}_{n}(p)=1 \text { for } p^{2} \leqq n^{2} ; \hat{h}_{n}(p)=0 \text { for } p^{2} \geqq(n+1)^{2} .
\end{gathered}
$$

Then $\Phi * h_{n} \in C^{\infty}$ for $\Phi \in \mathscr{S}^{\prime}$ and

$$
K_{n}\left(\Phi_{g}\right):=K\left(\left(\Phi * h_{n}\right)_{g}\right) \quad(n=1,2,3, \ldots)
$$

is a $\mathscr{C}_{4}$ operator on $\mathscr{H}$ for all $\Phi \in \mathscr{S}^{\prime}$ and it is obviously a weakly measurable function on $\mathscr{S}^{\prime}$. It is now easy to check that the $K_{n}\left(\Phi_{g}\right)$ form a Cauchy sequence in $\mathscr{C}_{4 ; 4}$ and therefore converge to a limit in $\mathscr{C}_{4 ; 4}$ which we denote by $K\left(\Phi_{g}\right)$, as $n \rightarrow \infty$. This, and the independence of the limit of the cutoff procedure, follows from the convergence of the Feynman graphs Fig. 1 and Fig. 2 (where - H denotes $\left.\frac{1}{\sqrt{p^{2}+m^{2}}}\right)$

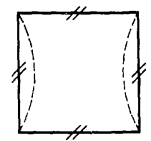

Fig. 1

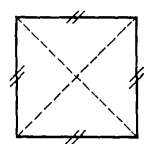

Fig. 2

which arise in the computation of $\|K\|_{4 ; 4}^{4}=\int \operatorname{Tr}\left(K K^{*}\right)^{2} d \mu_{0}$. For $A \in \mathscr{C}_{n+1}$ we now define (cf. $[14,24]$ )

$$
\operatorname{det}_{(n)}(1+A):=\operatorname{det}\left[(1+A) \exp \left(\sum_{k=1}^{n} \frac{1}{k}(-A)^{k}\right)\right] .
$$

The (norm convergent) power series for $B=(1+A) e^{\sum_{k=1}^{n} \frac{1}{k}(-A)^{k}}-1$ starts with $A^{n+1}$, that means $B=A^{n+1} D$ with some bounded operator $D$, which implies $B \in \mathscr{C}_{1}$. Therefore the right hand side of (2.7) makes sense (cf. Ruston [15]). Since for almost every $\Phi \in \mathscr{S}^{\prime} K \in \mathscr{C}_{4}$, $\operatorname{det}_{(3)}(1+\lambda K)$ is an almost everywhere

\footnotetext{
1 By this we mean that all matrix elements are measurable.
} 


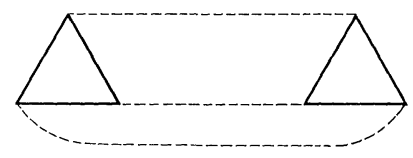

Fig. 3

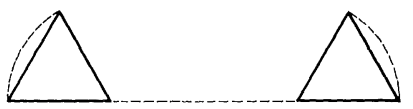

Fig. 4

finite random variable ${ }^{2}$. The same is true of $\operatorname{Tr} K^{3}$ [it is in $L^{2}\left(d \mu_{0}\right)$, because the Feynman graphs Figs. 3, 4 etc. are all finite]; this allows us to define

$$
\operatorname{det}_{(2)}(1+\lambda K):=\operatorname{det}_{(3)}(1+\lambda K) e^{\frac{\lambda^{3}}{3} \operatorname{Tr} K^{3}}
$$

as an a.e. finite random variable (the measurability is obvious).

$\operatorname{Tr} K^{2}$ is logarithmically divergent (it corresponds to the Feynman graph Fig. 5).

This forces us to introduce an infinite mass counterterm as to be expected from perturbation theory: We introduce an ultraviolet cutoff function $h_{\kappa_{f}}$ for the fermion propagator:

$$
h_{\kappa_{f}}(x)=\frac{1}{(2 \pi)^{2}} \int_{p^{2} \leqq \kappa_{f}^{2}} d^{2} p e^{i p x}
$$

and consider

where

$$
\operatorname{Tr}: K_{\kappa_{f}}^{2}:+\lambda^{-2} \delta \mu_{\kappa_{f}}^{2}: \Phi_{g}^{2}:=F_{\kappa_{f}}\left(\Phi_{g}\right)
$$

$$
K_{\kappa_{f}}=S_{F}{ }^{\left(\kappa_{f}\right)} \Gamma \Phi_{g} ; S_{F}^{\left(\kappa_{f}\right)}=S_{F} * h_{\kappa_{f}} .
$$

If we choose the $\kappa_{f}$-dependence of $\delta \mu_{\kappa_{f}}{ }^{2}$ suitably

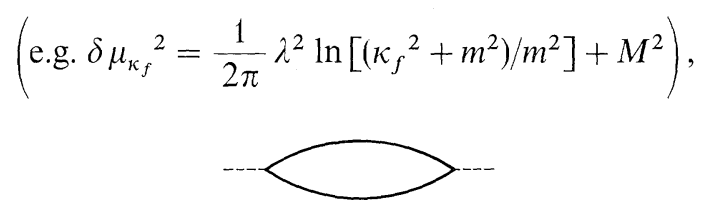

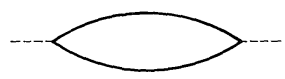

Fig. 5

this provides the necessary subtraction for the graph Fig. 5 (cf. Appendix A) and

$$
F_{\kappa_{f}}\left(\Phi_{g}\right) \rightarrow:\left(\Phi, B_{M} \Phi\right)_{1}:=\operatorname{Tr}_{\mathrm{reg}}: K^{2}:+\frac{M^{2}}{\lambda^{2}} \int: \Phi_{g}(x)^{2}: d x
$$

in $L^{2}\left(d \mu_{0}\right)$, where $B_{M}$ is a Hilbert-Schmidt operator on the real Hilbert space $\mathscr{H}_{1}$, the completion of $\mathscr{S}$ with respect to the scalar product $(f, g)_{1}=\left(f,\left(-\Delta+\mu^{2}\right) g\right)$. The Wick ordering in (2.10) is necessary because $B_{M}$ is not trace class (the Wick subtraction term is infinite). $B_{M}$ depends on the renormalization parameter $M$ which expresses the fact that the renormalized mass can be chosen arbitrarily and independently of the bare mass $\mu$.

\footnotetext{
${ }^{2}$ Measurability follows from the power series expansion in $\lambda$ (which is convergent for all $\lambda \in \mathbb{C}$ ).
} 
We are now ready to define

$$
\operatorname{det}_{\text {ren }}(1+\lambda K)=\operatorname{det}_{(2)}(1+\lambda K) e^{-\frac{\lambda^{2}}{2}:\left(\Phi, B_{M} \Phi\right)_{1}:} .
$$

If we compare (2.12) with the formal expression for $\operatorname{det}(1+\lambda K)$ we see that we have introduced three types of counterterms: A constant (hidden in the Wick ordering of : $\left.\left(\Phi, B_{M} \Phi\right)_{1}:\right)$, a term linear in $\Phi$ (since we dropped $\operatorname{Tr} K$ altogether) which corresponds to Wick ordering for the fermions, and a term quadratic in $\Phi$ (the boson mass counterterm) (2.12) defines an a.e. $\left(d \mu_{0}\right)$ finite random variable on $\mathscr{S}^{\prime 3}$.

Next we show

\section{Lemma 2.2.}

$$
\begin{array}{lll}
\operatorname{det}_{\text {ren }}\left(1+\lambda K\left(\Phi_{g}\right)\right)>0 & \text { for } & \Gamma=i \gamma_{5} \\
\operatorname{det}_{\text {ren }}\left(1+\lambda K\left(\Phi_{g}\right)\right) \geqq 0 & \text { for } & \Gamma=1
\end{array}
$$

Proof. First we show that $\operatorname{det}_{\text {ren }}$ is real for $\lambda \in \mathbb{R}$.

a) Scalar case $(\Gamma=1)$

$$
K=\frac{\not p+m}{p^{2}+m^{2}} \Phi_{g} ; K^{*}=\frac{1}{\sqrt{p^{2}+m^{2}}} \Phi_{g} \frac{-\not p+m}{\sqrt{p^{2}+m^{2}}} .
$$

With the unitary operator

$$
U=\frac{\not p+m}{\sqrt{p^{2}+m^{2}}} \gamma_{5}
$$

we get

$$
U K U^{-1}=K^{*}
$$

$\overline{\operatorname{det}_{(2)}(1+\lambda K)}=\operatorname{det}_{(2)}\left(1+\lambda K^{*}\right)=\operatorname{det}_{(2)}\left(1+\lambda U K U^{-1}\right)=\operatorname{det}_{(2)}(1+\lambda K)$.

$:\left(\Phi, B_{M} \Phi\right)_{1}$ : is obviously real.

b) Pseudoscalar case $\left(\Gamma=i \gamma_{5}\right)$

$$
K=\frac{\not p+m}{p^{2}+m^{2}} i \gamma_{5} \Phi_{g} ; K^{*}=-\frac{1}{\sqrt{p^{2}+m^{2}}} i \gamma_{5} \Phi_{g} \frac{-\not p+m}{\sqrt{p^{2}+m^{2}}} .
$$

There is a conjugation $j$ (antiunitary), such that

$$
j \gamma_{\mu} j=-\gamma_{\mu} ; j i \gamma_{5} j=i \gamma_{5}
$$

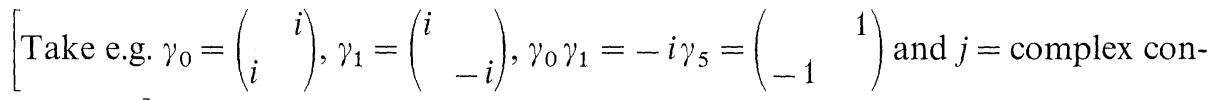
jugation.]

$\overline{\operatorname{det}_{(2)}(1+\lambda K)}=\operatorname{det}_{(2)}(1+\lambda j K j)=\operatorname{det}_{(2)}\left(1+\lambda \gamma_{5} j K j \gamma_{5}\right)=\operatorname{det}_{(2)}(1+\lambda K)$.

The reality of : $\left(\Phi, B_{M} \Phi\right)_{1}$ : is again clear.

${ }^{3}$ After completing this paper, I learned that Bellissard [16] also has established the renormalized determinant as an a.e. finite random variable. 
Remark. With the above $U(2.15)$ we have $U K U^{-1}=-K^{*}$, hence

$$
\operatorname{det}_{(2)}(1+\lambda K)=\operatorname{det}_{(2)}\left(1-\lambda K^{*}\right)=\operatorname{det}_{(2)}(1-\lambda K) .
$$

If we expand $\ln \operatorname{det}_{(2)}(l+\lambda K)$ for small $\lambda$, we see that

("Furry's theorem").

$$
\operatorname{Tr} K^{2 n+1}=0 \quad(n=1,2, \ldots)
$$

Now we turn to the positivity statements (2.13). It is sufficient to show

$$
\left.\begin{array}{ll}
F(\lambda)=\operatorname{det}_{(3)}(1+\lambda K)>0 & \text { for } \quad \Gamma=i \gamma_{5} \\
F(\lambda)=\operatorname{det}_{(3)}(1+\lambda K) \geqq 0 & \text { for } \quad \Gamma=1
\end{array}\right\} \lambda \in \mathbb{R} .
$$

$F(\lambda)$ is an entire function and $F(0)=1$. If (2.23) does not hold, there is a real $\lambda_{0}$ with $F\left(\lambda_{0}\right)=0$. Then $-\frac{1}{\lambda_{0}}$ is an eigenvalue of $K$ (see e.g. Brascamp [14]).

a) Pseudoscalar case

$$
\begin{gathered}
\text { Let } V=\frac{-\not p+m}{\sqrt{p^{2}+m^{2}}} \quad(V \text { unitary }) ; \\
\psi \in \mathscr{H},\|\psi\|=1 \\
\|(1+\lambda K) \psi\| \geqq|(\psi, V(1+\lambda K) \psi)| \geqq|\operatorname{Re}(\psi, V(1+\lambda K) \psi)|=\left(\psi, \frac{m}{\sqrt{p^{2}+m^{2}}} \psi\right)>0
\end{gathered}
$$
(since $V K+K^{*} V^{*}=0$ ).

This shows that $K$ has no real eigenvalues, therefore the first line of (2.23) is proved.

b) Scalar case

Here the situation seems to be different. We cannot prove the absence of real eigenvalues for $K=\frac{\not p+m}{p^{2}+m^{2}} \Phi_{g}$. But let us consider a modified operator

$$
K_{m^{\prime}}=\frac{\not p+m+i m^{\prime} \gamma_{5}}{p^{2}+m^{2}+m^{\prime 2}} \Phi_{g} \quad\left(m^{\prime}>0\right) .
$$

With $V=i \gamma_{5} \frac{-\not p+m-i \gamma_{5} m^{\prime}}{\sqrt{p^{2}+m^{2}+m^{\prime 2}}}$ and $\psi \in \mathscr{H},\|\psi\|=1$ we have

$$
\left\|\left(1+\lambda K_{m^{\prime}}\right) \psi\right\| \geqq\left|\operatorname{Re}\left(\psi, V\left(1+\lambda K_{m^{\prime}}\right) \psi\right)\right|=\left(\psi, \frac{m^{\prime}}{\sqrt{p^{2}+m^{2}+m^{\prime 2}}} \psi\right)>0
$$

hence

$$
\operatorname{det}_{(3)}\left(1+\lambda K_{m^{\prime}}\right)>0 \quad(\lambda \in \mathbb{R})
$$

[the reality of $\operatorname{det}_{(3)}\left(1+\lambda K_{m^{\prime}}\right)$ follows as in Eq. (2.23)].

If we let $m^{\prime} \rightarrow 0,(2.21)$ follows (the continuous dependence of $\operatorname{det}_{(3)}$ on $m^{\prime}$ is no problem; it actually follows from estimates of the type proved in the next section). 


\section{Integrability of the Renormalized Determinant}

This is the main point of this paper. Since the proof is rather complicated, let us first outline the strategy. We have

where

$$
\operatorname{det}_{\mathrm{ren}}(1+\lambda K)=u v
$$

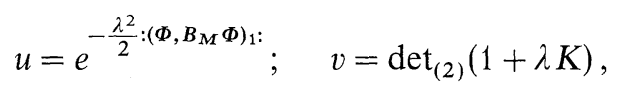

From $v$ we will extract a second Gaussian factor:

$$
v=v_{1} \cdot v_{2} ; \quad v_{1}=e^{\frac{\lambda^{2}}{2}:\left(\Phi, B_{1} \Phi\right)_{1}:}
$$

where $B_{1}$ again is Hilbert-Schmidt. For any given $q>1$ we can choose the finite mass renormalization parameter $M$ in such a way that

$$
u v_{1}=e^{-\frac{\lambda^{2}}{2}:\left(\Phi, B_{M} \Phi\right)_{1}:+\frac{\lambda^{2}}{2}:\left(\Phi, B_{1} \Phi\right)_{1}:}=e^{-\frac{\lambda^{2}}{2}:(\Phi, C \Phi)_{1}:} \in L^{q}\left(d \mu_{0}\right),
$$

$v_{2}$ will be shown to be in $\bigcap_{p<\infty} L^{p}$ hence $u v \in L^{q_{1}}$ for all $q_{1}<q$. Now let us turn to $v=\operatorname{det}_{(2)}(1+\lambda K)$. To estimate $v$, we proceed in a similar way as Nelson $[11,17]$ in his proof for $P(\Phi)_{2}$ theories. We introduce an ultraviolet cutoff function $h_{\kappa}(x)$ $\frac{1}{(2 \pi)^{2}} \int_{p^{2} \leqq \kappa^{2}} d^{2} p e^{i p x} \in C^{\infty}$ and study the behavior of

$$
v_{\kappa}=\operatorname{det}_{(2)}\left(1+\lambda K\left(h_{\kappa} * \Phi\right)_{g}\right)
$$

for large $\kappa\left(h_{\kappa}(x) \rightarrow \delta(x), \kappa \rightarrow \infty\right)$. We should like to estimate $\left|\ln v-\ln v_{\kappa}\right|$ by something like a polynomial in $\Phi$ which goes to zero fast enough for $\kappa \rightarrow \infty$. The problem is that $v$ or $v_{\kappa}$ might become small when eigenvalues of $K\left(\Phi_{g}\right)$ or $\left(K\left(h_{\kappa} * \Phi\right)_{g}\right)$ come close to the real axis. But this should not affect the integrability. Therefore we "cut out" the regions in $\mathscr{S}^{\prime}$ where this might happen.

We define for $\lambda \in \mathbb{R}$

Then

$$
\begin{aligned}
A & :=\lambda K+\lambda K^{*}+\lambda^{2} K^{*} K, \\
A_{\kappa} & :=\lambda K_{\kappa}+\lambda K_{\kappa}{ }^{*}+\lambda^{2} K_{\kappa}{ }^{*} K_{\kappa} \\
\left(K_{\kappa} K_{\kappa}\right. & \left.=K\left(\left(\Phi * h_{\kappa}\right)_{g}\right)\right) .
\end{aligned}
$$

$$
\begin{aligned}
\ln v^{2}= & \ln \left(\operatorname{det}_{(2)}(1+\lambda K)\right)^{2} \\
& =\ln \operatorname{det}_{(3)}(1+A)-\frac{\lambda^{4}}{2} \operatorname{Tr}\left(K K^{*}\right)^{2}-2 \lambda^{3} \operatorname{Tr} K^{2} K^{*}+\frac{1}{3} \operatorname{Tr} A^{3} .
\end{aligned}
$$

By $A_{+}, A_{\kappa+}$ we denote the positive parts (including 0 ) of the selfadjoint $\mathscr{C}_{4^{-}}$ operators $A, A_{\kappa}$, respectively. Then

$$
\begin{aligned}
\ln \operatorname{det}_{(3)}(1+A) & \leqq \ln \operatorname{det}_{(3)}\left(1+A_{+}\right) \\
& \leqq \ln \operatorname{det}_{(3)}\left(1+A_{\kappa+}\right)+\left|\ln \operatorname{det}_{(3)}\left(1+A_{\kappa+}\right)-\ln \operatorname{det}_{(3)}\left(1+A_{+}\right)\right| .
\end{aligned}
$$


(The first inequality in (3.9) follows from $\ln (1+x)-x+\frac{x^{2}}{2}-\frac{x^{3}}{3} \leqq 0$ for $-1<x<\infty$.) What we now need is an estimate for

$$
\left|\ln \operatorname{det}_{(3)}\left(1+A_{\kappa+}\right)-\ln \operatorname{det}_{(3)}\left(1+A_{+}\right)\right| .
$$

This is provided by the following two lemmas:

Lemma 3.1. Let $C, D \in \mathscr{C}_{4} ; C, D \geqq 0$. We denote the eigenvalues of $C$ and $D$ by $\lambda_{k}$, $\mu_{k}$ respectively and assume $\lambda_{1} \geqq \lambda_{2} \geqq \cdots \geqq 0 ; \mu_{1} \geqq \mu_{2} \geqq \cdots \geqq 0$. Then

$$
\begin{aligned}
d & =\left|\ln \operatorname{det}_{(3)}(1+C)-\ln \operatorname{det}_{(3)}(1+D)\right| \\
& \leqq\left(\sum_{k}\left|\lambda_{k}-\mu_{k}\right|^{4}\right)^{\frac{1}{4}} \sum_{l=0}^{3} c_{l}\|C\|_{4}^{l}\|D\|_{4}^{3-l}
\end{aligned}
$$

with some constants $c_{l}$.

Proof. We put $v_{k}(t)=t \lambda_{k}+(1-t) \mu_{k}(k=1,2,3, \ldots ; t \in[0,1])$.

$$
\begin{aligned}
d= & \mid \sum_{k} \ln \left(1+\lambda_{k}\right)-\lambda_{k}+\frac{1}{2} \lambda_{k}^{2}-\frac{1}{3} \lambda_{k}^{3} \\
& -\ln \left(1+\mu_{k}\right)+\mu_{k}-\frac{1}{2} \mu_{k}^{2}+\frac{1}{3} \mu_{k}^{3} \mid \\
= & \left|\sum_{k} \int_{0}^{1} d t \frac{d}{d t}\left(\ln \left(1+v_{k}(t)\right)-v_{k}(t)+\frac{1}{2} v_{k}(t)^{2}-\frac{1}{3} v_{k}(t)^{3}\right)\right| \\
\leqq & \sum_{k} \int_{0}^{1} d t\left|\frac{\lambda_{k}-\mu_{k}}{1+v_{k}(t)} v_{k}(t)^{3}\right| \\
\leqq & \sum_{k} \int_{0}^{1} d t\left|\lambda_{k}-\mu_{k}\right|\left|v_{k}(t)\right|^{3} \\
\leqq & \left(\sum_{k}\left|\lambda_{k}-\mu_{k}\right|^{4}\right)^{\frac{1}{4}} \int_{0}^{1}\left[t\left(\sum_{k} \lambda_{k}^{4}\right)^{\frac{1}{4}}+(1-t)\left(\sum_{k} \mu_{k}^{4}\right)^{\frac{1}{4}}\right] 3 d t \\
= & \left(\sum_{k}\left|\lambda_{k}-\mu_{k}\right|^{4}\right)^{\frac{1}{4}} \sum_{l=0}^{3} c_{l}\|C\|_{4}^{l}\|D\|_{4}^{3-l} .
\end{aligned}
$$

(We have used Hölder's inequality in the next to last step.)

Lemma 3.2. (Generalization of a theorem of Lidskii; see Kato [18], II., Theorems 6.10 and 6.11.) Let $C, D$ be selfadjoint $\mathscr{C}_{p}$-operators with eigenvalues $\lambda_{k}$, $\mu_{k}$ respectively $(k=1,2,3, \ldots)$. We define

$$
\lambda_{k}^{+}=\frac{1}{2}\left(\lambda_{k}+\left|\lambda_{k}\right|\right) ; \quad \mu_{k}^{+}=\frac{1}{2}\left(\mu_{k}+\left|\mu_{k}\right|\right) \quad(k=1,2, \ldots)
$$

and assume that the $\lambda_{k}{ }^{+}, \mu_{k}{ }^{+}$form a decreasing sequence. Then

$$
\sum_{k}\left|\lambda_{k}^{+}-\mu_{k}^{+}\right|^{p} \leqq\|C-D\|_{p}^{p}
$$

Proof. We approximate $C, D$ in the $p$-norm by finite rank operators $C_{N}, D_{N}$ in the following standard way. Let $P_{N}$ be the projection onto the subspace corre- 
sponding to eigenvalues $\left|\lambda_{k}\right| \geqq \frac{1}{N}$; then we take $C_{N}=P_{N} C . D_{N}$ is defined analogously. According to an easy application of Lidskii's theorem (see [16])

$$
\sum_{k}\left|\lambda_{k}^{(N)}-\mu_{k}^{(N)}\right|^{p} \leqq\left\|C_{N}-D_{N}\right\|_{p}^{p},
$$

where $\lambda_{k}^{(N)}, \mu_{k}^{(N)}$ are the eigenvalues of $C_{N}, D_{N}$, respectively. The inequality remains true if we replace the left hand side by $\Sigma\left|\lambda_{k}{ }^{+(N)}-\mu_{k}{ }^{+(N)}\right|^{p}$. If we let $N \rightarrow \infty$ the assertion (3.12) follows. By combining the two lemmas we get

$$
\begin{aligned}
& \left|\ln \operatorname{det}_{(3)}\left(1+A_{+}\right)-\ln \operatorname{det}_{(3)}\left(1+A_{\kappa+}\right)\right| \\
& \quad \leqq\left\|A-A_{\kappa}\right\|_{4} \sum_{l=0}^{3} c_{l}\|A\|_{4}^{l}\left\|A_{\kappa}\right\|_{4}^{3-l} .
\end{aligned}
$$

We note that

$$
\begin{aligned}
\ln \operatorname{det}_{(3)}\left(1+A_{\kappa+}\right) & \leqq \frac{1}{2} \operatorname{Tr} A_{\kappa+}{ }^{2}-\frac{1}{3} \operatorname{Tr} A_{\kappa+}{ }^{3} \\
& \leqq \frac{1}{2} \operatorname{Tr} A_{\kappa}{ }^{2}-\frac{1}{3} \operatorname{Tr} A_{\kappa}{ }^{3}
\end{aligned}
$$

Combining (3.8), (3.9), (3.14), and (3.15) we get

$$
\begin{aligned}
& \ln \left(\operatorname{det}_{(2)}(1+\lambda K)\right)^{2} \leqq \frac{1}{2} \operatorname{Tr} A_{\kappa}{ }^{2}-\frac{1}{3} \operatorname{Tr} A_{\kappa}{ }^{3} \\
&-\frac{\lambda^{4}}{2} \operatorname{Tr}\left(K K^{*}\right)^{2}-2 \lambda^{3} \operatorname{Tr} K^{2} K^{*}+\frac{1}{3} \operatorname{Tr} A^{3} \\
& \quad+\left\|A-A_{\kappa}\right\|_{4} \sum_{k=0}^{3} c_{k}\|A\|_{4}^{k}\left\|A_{\kappa}\right\|_{4}^{3-k}
\end{aligned}
$$

or

$$
\begin{aligned}
\ln \left(\operatorname{det}_{(2)}(1+\lambda K)\right)^{2} \leqq & \frac{\lambda^{2}}{2} \operatorname{Tr}\left(K_{\kappa}+K_{\kappa}^{*}\right)^{2} \\
& +\frac{\lambda^{4}}{2} \operatorname{Tr}\left(\left(K_{\kappa} K_{\kappa}^{*}\right)^{2}-\left(K K^{*}\right)^{2}\right)+2 \lambda^{3} \operatorname{Tr}\left(K_{\kappa}{ }^{2} K_{\kappa}^{*}-K^{2} K^{*}\right) \\
& +\frac{1}{3} \operatorname{Tr}\left(A^{3}-A_{\kappa}^{3}\right)+\left\|A-A_{\kappa}\right\|_{4} \sum_{k=0}^{3} c_{k}\|A\|_{4}^{k}\left\|A_{\kappa}\right\|_{4}^{3-k}
\end{aligned}
$$

The first term on the right hand side needs further discussion: For $\kappa \rightarrow \infty$

$$
\frac{1}{2} \operatorname{Tr}:\left(K_{\kappa}+K_{\kappa}^{*}\right)^{2}: \rightarrow:\left(\Phi, B_{1} \Phi\right)_{1}: \quad \text { in } \quad L^{2}\left(d \mu_{0}\right),
$$

where $B_{1}$ is a Hilbert-Schmidt operator on $\mathscr{H}_{1}$. For some constants $c, \kappa_{0}$ and large enough $\kappa$ we have

$$
\int \frac{1}{2} \operatorname{Tr}\left(K_{\kappa}+K_{\kappa}^{*}\right)^{2} d \mu_{0} \leqq c \ln \frac{\kappa}{\kappa_{0}}
$$

(see Appendix A for details). We define now

$$
\ln v_{2}:=\ln \operatorname{det}_{(2)}(1+\lambda K)-\frac{\lambda^{2}}{2}:\left(\Phi, B_{1} \Phi\right)_{1}: .
$$


From (3.17)-(3.20) we get for $v_{2}$ the estimate

$$
\begin{aligned}
\ln v_{2}^{2} \leqq & c \lambda^{2} \ln \frac{\kappa}{\kappa_{0}}+\frac{\lambda^{2}}{2}\left[\operatorname{Tr}:\left(K_{\kappa}+K_{\kappa}^{*}\right)^{2}\right. \\
& \left.-:\left(\Phi, B_{1} \Phi\right)_{1}:\right]+\frac{\lambda^{4}}{2}\left(\operatorname{Tr}\left(K_{\kappa} K_{\kappa}^{*}\right)^{2}-\left(K K^{*}\right)^{2}\right) \\
& +2 \lambda^{3} \operatorname{Tr}\left(K_{\kappa}{ }^{2} K_{\kappa}^{*}-K^{2} K^{*}\right)+\frac{1}{3} \operatorname{Tr}\left(A^{3}-A_{\kappa}{ }^{3}\right) \\
& +\left\|A-A_{\kappa}\right\|_{4} \sum_{k=0}^{3} c_{k}\|A\|_{4}^{k}\left\|A_{\kappa}\right\|_{4}^{3-k} .
\end{aligned}
$$

This can be summarized in the following form:

$$
\begin{aligned}
\ln v_{2}^{2} \leqq & c \lambda^{2} \ln \frac{\kappa}{\kappa_{0}}+\sum_{r=1}^{N}\left|P_{r}(\Phi)-P_{r}\left(\Phi_{\kappa}\right)\right|^{\frac{\lambda_{r}}{4}} \\
& \cdot\left|Q_{r}(\Phi)\right|^{4}\left|R_{r}\left(\Phi_{\kappa}\right)\right|^{4} \quad\left(\lambda_{r} \in\{1,4\}\right)
\end{aligned}
$$

where $P_{r}, Q_{r}, R_{r}$ are (nonlocal) polynomials in $\Phi$ (or $\Phi_{\kappa}=\Phi * h_{\kappa}$ ) of degree at most 24 . They have the important property (which can be seen by looking at the corresponding Feynman graphs which all converge like a negative power of the cutoff as can be seen by simple power counting): There is a $\varepsilon>0$ such that

$$
\begin{aligned}
\int\left|P_{r}(\Phi)-P_{r}\left(\Phi_{\kappa}\right)\right|\left|Q_{r}(\Phi) R_{r}\left(\Phi_{\kappa}\right)\right| & d \mu_{0} \\
& \leqq\left\|P_{r}(\Phi)-P_{r}\left(\Phi_{\kappa}\right)\right\|_{2}\left\|Q_{r}(\Phi) R_{r}\left(\Phi_{\kappa}\right)\right\|_{2} \\
& =O\left(\kappa^{-\varepsilon}\right) \quad(r=1, \ldots, N) .
\end{aligned}
$$

We can therefore apply Nelson's reasoning [15]: By hypercontractivity for $p \geqq 1$ we have

$$
\left\|P_{r}(\Phi)-P_{r}\left(\Phi_{\kappa}\right)\right\|_{p}^{p / 2}\left\|Q_{r}(\Phi) R_{r}\left(\Phi_{\kappa}\right)\right\|_{p}^{p / 2} \leqq C \kappa^{-\varepsilon p}(p-1)^{2 p}
$$

for some $C, \varepsilon>0$ and therefore (cf. Nelson [17])

$$
\mu_{0}\left\{\left|P_{r}(\Phi)-P_{r}\left(\Phi_{\kappa}\right)\right|\left|Q_{r}(\Phi) R_{r}\left(\Phi_{\kappa}\right)\right| \geqq 1\right\} \leqq e^{-b \kappa^{-\varepsilon}}
$$

for some $b, \varepsilon>0$. This implies as in Nelson's case

$$
v_{2} \in L^{p}\left(d \mu_{0}\right) \text { for } p \in[1, \infty) \text {. }
$$

We now have to investigate the Gaussian factor $u v_{1}$ (3.4). We need the following rather obvious (and perhaps well known)

Lemma 3.3. Let $C=C^{*}$ be a Hilbert-Schmidt operator on $\mathscr{H}_{1} \cdot u=e^{-\frac{1}{2}:(\Phi, C \Phi)_{1}:} \in L^{p}$ if and only if $1+p C>0(p \geqq 1)$.

Proof. Assume $1+p C>0$. By introducing a suitable orthonormal basis $\left(e_{n}\right)_{n=1}^{\infty}$ in $\mathscr{H}_{1}, C$ can be diagonalized $\left(\Phi_{n}=\left(\Phi, e_{n}\right)_{1}\right)$

$$
:(\Phi, C \Phi)_{1}:=\sum_{n=1}^{\infty} \gamma_{n}: \Phi_{n}{ }^{2}:=\sum_{n=1}^{\infty} \gamma_{n}\left(\Phi_{n}{ }^{2}-1\right) \text {. }
$$


We approximate $u$ by

$$
u_{N}:=\exp \left(-\frac{1}{2} \sum_{n=1}^{N} \gamma_{n}\left(\Phi_{n}{ }^{2}-1\right)\right) .
$$

Since $\ln u_{N} \rightarrow \ln u$ in $L^{2}\left(d \mu_{0}\right)$, there is a subsequence $u_{N_{k}}$ converging to $u$ a.e. $\left(d \mu_{0}\right)$.

$$
\begin{aligned}
\left\|u_{N}\right\|_{p}^{p} & =\prod_{n=1}^{N} \frac{1}{\sqrt{2 \pi}} \int_{-\infty}^{\infty} d \Phi_{n} e^{-\frac{1}{2} \Phi_{n}^{2}-\frac{p}{2} \gamma_{n} \Phi_{n}^{2}+\frac{1}{2} \gamma_{n}} \\
& =\prod_{n=1}^{N} \frac{1}{\sqrt{1+p \gamma_{n}}} e^{\frac{1}{2} p \gamma_{n}} \rightarrow \prod_{n=1}^{\infty} \frac{1}{\sqrt{1+p \gamma_{n}}} e^{\frac{1}{2} p \gamma_{n}} \\
& \leqq \exp \left(\frac{1}{2} \sum_{n=1} \frac{p^{2} \gamma_{n}^{2}}{1+p \gamma_{n}}\right)<\infty .
\end{aligned}
$$

By Fatou's lemma $u \in L^{p}$. The converse statement is trivial.

In our case

$$
\begin{aligned}
:(\Phi, C \Phi): & =\lambda^{2}:\left(\Phi,\left(B_{M}-B_{1}\right) \Phi\right)_{1}: \\
& =-\lambda^{2} \operatorname{Tr}_{\mathrm{reg}}: K K^{*}:+M^{2} \int: \Phi_{g}(x)^{2}: d^{2} x
\end{aligned}
$$

Therefore $C$ has the form (cf. Appendix A)

$$
C=\frac{2}{k^{2}+\mu^{2}} g\left(-\lambda^{2} G_{\mathrm{reg}}+\frac{1}{2} M^{2}\right) g,
$$

where $G_{\text {reg }}$ is a multiplication operator in momentum space $[g$ denotes multiplication by $g(x)$ in configuration space]. $G_{\text {reg }}$ is bounded from above

$$
G_{\text {reg }}(k) \leqq \frac{1}{2} a<\infty^{4}
$$

[see Appendix A, Eqs. (A.12) and (A.14)], therefore

$$
C \geqq \frac{1}{p^{2}+\mu^{2}} g\left(-a \lambda^{2}+M^{2}\right) g .
$$

In order to have $u v_{1}(3.4)$ in $L^{r}(r \geqq 1)$ it suffices according to Lemma 3.3 if

$$
(\psi, \psi)_{1}+(g \psi, g \psi)_{0}\left(-a \lambda^{2}+M^{2}\right) r>0
$$

for each $\psi \in \mathscr{H}_{1}\left((,)_{0}\right.$ denotes the scalar product of $\left.L^{2}\left(d^{2} x\right)\right)$. For $0 \leqq g \leqq 1$ ( $g$ measurable)

$$
(g \psi, g \psi)_{0} \leqq(\psi, \psi)_{0} \leqq \frac{1}{\mu^{2}}(\psi, \psi)_{1} .
$$

Therefore (3.34) is fulfilled, if we choose

$$
M^{2}>a \lambda^{2}-\frac{\mu^{2}}{r}
$$

\footnotetext{
4 This is actually true for $a=0$.
} 
Let us summarize: For every given $q \geqq 1$ there is a choice of $M^{2}$ such that $\operatorname{det}_{\text {ren }}(1+\lambda K) \in L^{q}$. Actually we have shown that

$$
\operatorname{det}_{\text {ren }}(1+\lambda K) \leqq w_{M}(\Phi) \in L^{q}
$$

for suitable $M=M(q)$, where

$$
\begin{aligned}
w_{M}(\Phi)^{2}= & e^{-\lambda^{2}:\left(\Phi, B_{M} \Phi\right):} \operatorname{det}_{(3)}\left(1+A_{+}\right) \\
& \cdot \exp \left\{-\frac{\lambda^{4}}{2} \operatorname{Tr}\left(K K^{*}\right)^{2}-2 \lambda^{3} \operatorname{Tr} K^{2} K^{*}+\frac{1}{3} \operatorname{Tr} A^{3}\right\}
\end{aligned}
$$

\section{The Renormalized Matthews-Salam Formulae for the Schwinger Functions}

We now come back to formula (1.1) for the Schwinger functions

$$
\begin{aligned}
& S^{\prime}\left(x_{1}, \ldots, x_{n} ; y_{1}, \ldots, y_{m} ; z_{1}, \ldots, z_{m}\right) \\
& \quad=\frac{1}{Z(\lambda)} \int d \mu_{0} \prod_{l=1}^{n} \Phi\left(x_{l}\right) \operatorname{det}_{i k} S_{F}{ }^{\prime}\left(y_{i}, z_{i} ; \Phi_{g}\right) \operatorname{det}_{\mathrm{ren}}\left(1+\lambda K\left(\Phi_{g}\right)\right) .
\end{aligned}
$$

In Section 3 we showed that $\operatorname{det}_{\text {ren }}(1+\lambda K) \in L^{1}$ (for suitable choice of $M$ ), hence $Z(\lambda)<\infty(\lambda \in \mathbb{R})$. The next question is whether $Z(\lambda)$ might vanish. The answer is

Lemma 4.1.

$$
Z(\lambda)=\int \operatorname{det}_{\text {ren }}(1+\lambda K) d \mu_{0} \neq 0 \quad \text { for } \quad \lambda \in \mathbb{R} .
$$

Proof. $Z(\lambda)=0$ implies

$$
\operatorname{det}_{\text {ren }}(1+\lambda K)=0
$$

for almost all $\Phi \in \mathscr{S}^{\prime}$ and therefore

for almost all $\Phi \in \mathscr{S}^{\prime}$.

$$
\operatorname{det}_{(3)}(1+\lambda K)=0
$$

In the pseudoscalar case this is excluded by (2.23).

This simple argument does not work in the scalar case. But we remark that for all $\Phi \in \mathscr{S}^{\prime}$ for which $\|\lambda K\|<1$

$$
\operatorname{det}_{(3)}(1+\lambda K) \neq 0
$$

[and hence $>0$ by $(2.23)]$.

Since $\|K\| \leqq\|K\|_{4}$, it suffices to show that the set of $\Phi \mathscr{S}^{\prime}$ for which $\|\lambda K\|_{4}<1$ has nonvanishing measure. This follows from

Lemma 4.2. $\mu_{0}\left\{\|K\|_{4}^{4}<\varepsilon\right\}>0$ for all $\varepsilon>0$.

The proof of this lemma, which is not quite as trivial as one might expect, follows from a more general statement about monomials in the free field which is proven in Appendix B.

Next we discuss the numerator of (4.1). Of course we have to smear with suitable test functions. We prove

\section{Lemma 4.3.}

$$
\begin{aligned}
\left|\operatorname{det}_{i k} S_{F}^{\prime}\left(f_{i}, g_{k} ; \Phi_{g}\right)\right| \operatorname{det}_{\mathrm{ren}}(1+\lambda K) & \leqq \prod_{i=1}^{m}\left\|f_{i}\right\|_{\mathscr{H}^{*}}\left\|g_{i}\right\|_{\mathscr{H}^{*}} e^{11 m / 12} w_{M}(\Phi),
\end{aligned}
$$


where $w_{M}$ is given by (3.37), and \|\|$_{\mathscr{H} *}$ denotes the norm of the Hilbert space $\mathbb{C}^{2} \otimes \mathscr{H}_{-\frac{1}{2}}$, the dual of $\mathscr{H}(2.1)$ (the first factor on the left hand side does not necessarily have an independent meaning for all $\lambda$ in the scalar case).

Proof. Let $f_{i}, g_{i} \in \mathscr{H}^{*}(i=1, \ldots, m)$. According to (1.3)

$$
\begin{aligned}
S_{F}{ }^{\prime}\left(f_{i}, g_{k} ; \Phi_{g}\right) & =\left(f_{i},(1+\lambda K)^{-1} S_{F} g_{k}\right)_{0} \\
& =\left(\frac{1}{\sqrt{p^{2}+m^{2}}} f_{i},(1+\lambda K)^{-1} S_{F} g_{k}\right)_{\mathscr{H}}
\end{aligned}
$$

$\left((,)_{0}\right.$ denotes the scalar product of $\mathbb{C}^{2} \otimes L^{2}\left(d^{2} x\right),(,)_{\mathscr{H}}$ the scalar product of $\left.\mathscr{H}\right)$.

Since in the scalar case we cannot exclude the possibility of real eigenvalues, let us consider the product

$$
\begin{gathered}
X=\left|\operatorname{det}_{i k}\left(\frac{1}{\sqrt{p^{2}+m^{2}}} f_{i}, \frac{1}{1+\lambda K} S_{F} g_{k}\right)\right|_{\mathscr{H}}^{2} \operatorname{det}_{(3)}(1+A) \\
\left(A=\lambda K+\lambda K^{*}+\lambda^{2} K^{*} K\right) .
\end{gathered}
$$

We note that $S_{F}=\frac{\not p+m}{p^{2}+m^{2}}$ and $D=\frac{1}{\sqrt{p^{2}+m^{2}}}$ (the duality map) are isometries from $\mathscr{H}^{*}$ onto $\mathscr{H}$. If we define

$$
\varphi=D f_{1} \wedge \cdots \wedge D f_{m} ; \psi=S_{F} g_{1} \wedge \cdots \wedge S_{F} g_{m}
$$

$\varphi, \psi \in \wedge^{m} \mathscr{H}(m$-fold antisymmetric tensor product of $\mathscr{H}$ ) we can rewrite $(4.8)$ and obtain

$$
\begin{aligned}
X & =\left|\left(\varphi,\left(\otimes^{m} \frac{1}{1+\lambda K}\right) \psi\right) \wedge^{m} \mathscr{H}\right|^{2} \operatorname{det}_{(3)}(1+A) \\
& \leqq(\varphi, \varphi)_{\Lambda} m_{\mathscr{H}}\left(\psi,\left(\otimes^{m} \frac{1}{1+A}\right) \psi\right) \wedge^{m} \mathscr{H} \operatorname{det}_{(3)}(1+A) ;
\end{aligned}
$$

(we choose the normalization as in Greub [19]). We now split $A$ :

$$
A=A_{+}-A_{-} ; A_{+} \geqq 0, A_{-}>0
$$

and denote the eigenvalues of $A_{-}$by $\alpha_{k}\left(1 \geqq \alpha_{1} \geqq \alpha_{2} \geqq \cdots\right)$.

$$
\begin{aligned}
X \leqq & (\varphi, \varphi)_{\Lambda} m_{\mathscr{H}}\left(\psi,\left(\otimes^{m} \frac{1}{1-A}\right) \psi\right) \wedge^{m} \mathscr{H} \operatorname{det}_{(3)}\left(1-A_{-}\right) \\
& \cdot \operatorname{det}_{(3)}\left(1+A_{+}\right) \\
\leqq & \|\varphi\|_{\Lambda^{m} \mathscr{H}}^{2}\|\psi\|_{\Lambda^{m} \mathscr{H}}^{2} \operatorname{det}_{(3)}\left(1+A_{+}\right) \\
& \cdot \prod_{l \geqq m+1}\left(1-\alpha_{l}\right) e^{\alpha_{l}+\frac{1}{2} \alpha_{l}{ }^{2}+\frac{1}{3} \alpha_{l}{ }^{3}} \prod_{l=1}^{m} e^{\alpha_{l}+\frac{1}{2} \alpha_{l}{ }^{2}+\frac{1}{3} \alpha_{l}{ }^{3}}
\end{aligned}
$$

If we use the fact

$$
(1-x) e^{x+x^{3} / 2+x^{3} / 3} \leqq 1(x \geqq 0)
$$

and the Def. (4.9), we get

$$
X \leqq \prod_{i=1}^{m}\left\|f_{i}\right\|_{\mathscr{H} *}^{2}\left\|g_{i}\right\|_{\mathscr{H} * \operatorname{det}_{(3)}}^{2}\left(1+A_{+}\right) e^{m\left(1+\frac{1}{2}+\frac{1}{3}\right)}
$$


Using the relation between $\operatorname{det}_{(3)}(1+A)$ and $\operatorname{det}_{\mathrm{ren}}(1+\lambda K)[(3.1)$, (3.2), (3.8)] and the definition of $w_{M}$ (3.37), we get the assertion (4.6). Since $\prod_{i=1}^{n} \Phi\left(h_{i}\right) \in \bigcap_{1 \leqq p<\infty} L^{p}\left(d \mu_{0}\right)$ for $h_{i} \in \mathscr{H}_{-1}(i=1, \ldots, n)$, we have shown that for suitably chosen $M$ the definition

$$
\begin{aligned}
& S\left(h_{1}, \ldots, h_{n} ; f_{1}, \ldots, f_{m} ; g_{1}, \ldots, g_{m}\right) \\
& \quad=\frac{1}{Z(\lambda)} \int d \mu_{0} \operatorname{det}_{i k} S_{F}{ }^{\prime}\left(f_{i}, g_{k} ; \Phi_{g}\right) \operatorname{det}_{\mathrm{ren}}(1+\lambda K) \prod_{r=1}^{n} \Phi\left(h_{r}\right)
\end{aligned}
$$

makes sense.

Because of

$$
\left\|\prod_{r=1}^{n} \Phi\left(h_{r}\right)\right\|_{p} \leqq(p-1)^{\frac{n}{2}}\left\|\prod_{r=1}^{n} \Phi\left(h_{r}\right)\right\|_{2}
$$

(see Nelson [11]) and

$$
\left\|\prod_{r=1}^{n} \Phi\left(h_{r}\right)\right\|_{2}^{2} \leqq \prod_{r=1}^{n}\left\|h_{r}\right\|_{-1}^{2} 2^{n} \frac{1}{\sqrt{\pi}} \Gamma\left(n+\frac{1}{2}\right)
$$

we get the following bound:

$$
\begin{aligned}
& \left|S\left(h_{1}, \ldots, h_{n} ; f_{1}, \ldots, f_{m} ; g_{1}, \ldots, g_{m}\right)\right| \\
& \quad \leqq c_{1} e^{c_{2} n+c_{3} m}\left(\Gamma\left(n+\frac{1}{2}\right)\right)^{\frac{1}{2}} \prod_{r=1}^{n}\left\|h_{r}\right\|_{-1} \prod_{i=1}^{m}\left\|f_{i}\right\|_{\mathscr{H} \mathscr{H}^{*}}\left\|g_{i}\right\|_{\mathscr{H} \mathcal{F}^{*}}
\end{aligned}
$$

with some constants $c_{1}, c_{2}, c_{3}$.

\section{Remarks}

Osterwalder and Schrader [5] have proved a Feynman-Kac formula for Boson-Fermion models with ultraviolet and volume cutoff, as mentioned earlier. From this formula and the integrability of the renormalized Fredholm determinant without cutoff it follows that

$$
\left(\Omega_{0}, e^{-H_{\kappa, V} t} \Omega_{0}\right)_{\mathscr{F}}=\int d \mu_{0} \operatorname{det}_{\mathrm{ren}}\left(1+\lambda K_{\kappa}(\Phi)\right)
$$

stays bounded for $\kappa \rightarrow \infty$ ( $\Omega_{0}$ is the Fock vacuum, $H_{\kappa, V}$ the doubly cutoff Hamiltonian with interaction in the volume $V, g$ the characteristic function of $V x[0, t])$.

Since we have actually shown that $\operatorname{det}_{\text {ren }}\left(1+\lambda K\left(\Phi_{g}\right)\right) \in L^{q}$ with $q>1$ (for suitable choice of the renormalization parameter $M), \int\left|\operatorname{det}_{\text {ren }}\left(1+\lambda K_{\kappa}\left(\Phi_{g}\right)\right)\right|^{1+\varepsilon} d \mu_{0}$ stays bounded for some $\varepsilon>0$ and $\kappa \rightarrow \infty$. By a criterion for "uniform integrability" (see for instance the appendix of Doob [20] $)^{5}$ it then follows that the limit of the integral equals the integral of the limit, that is

$$
\left(\Omega_{0}, e^{-H_{V} t} \Omega_{0}\right)_{\mathscr{F}}=\int d \mu_{0} \operatorname{det}_{\mathrm{ren}}\left(1+\lambda K\left(\Phi_{g}\right)\right) .
$$

\footnotetext{
${ }^{5} \mathrm{I}$ am indebted to H. Ezawa and J. R. Klauder for bringing this criterion to my attention.
} 
An analogous result is true for more general Fock space states $\psi_{0}, \chi_{0}$ which are created from $\Omega_{0}$ by applying polynomials in time zero Bose fields. By using Nelson's hypercontractivity (see $[11,12]$ ) it is easy to show that for large enough $t$

$$
\left|\left(\Psi_{0}, e^{-H_{V} t} \chi_{0}\right)_{\mathscr{F}}\right| \leqq\left\|\psi_{0}\right\|\left\|\chi_{0}\right\| C(V, t)
$$

which expresses boundedness below of $H_{V}$ for a "pure boson" subtheory. But in order to show boundedness below of $H_{V}$ on the full Fock space from our results it seems to be necessary to take the infinite time limit. It would be desirable, in any case, to perform the Euclidean infinite volume limit for the Schwinger functions and to check Osterwalder's and Schrader's axioms which then would imply all the Wightman axioms for the corresponding quantum field theory. Unfortunately the methods which were so successful in $P(\Phi)_{2}$ models like the cluster expansion of Glimm, Jaffe and Spencer [21] or the method of Dimock and Glimm [22] are not directly applicable, since the effective interaction for the bosons is nonlocal. Nevertheless the coupling between distant regions should fall off exponentially and this fact should lead to the existence of the infinite volume limit.

Acknowledgements. I thank A. Wightman for bringing the problem to my attention and I. Herbst for reading the manuscript. I profited from discussions with P. Federbush, I. Herbst, K. Osterwalder, B. Simon, and A. Wightman on the subject of this paper. Furthermore, I should like to thank the Institute for Advanced Study for the hospitality extended to me.

\section{Appendix}

\section{A. Computation of the Feynman Integrals Corresponding to $\operatorname{Tr} K^{2}$ and $\operatorname{Tr} K K^{*}$}

We want to prove Eqs. (3.18) and (3.19). Formally we have

$$
\begin{aligned}
\frac{1}{2} \operatorname{Tr} K^{2}{ }_{k} & =\int \Phi_{g, k}(x) \Phi_{g, k}(y) \operatorname{Tr} \Gamma S_{F}(x-y) \Gamma S_{F}(y-x) d^{2} x d^{2} y \\
& =\int d^{2} k\left|\tilde{\Phi}_{g, k}(k)\right|^{2} \int d^{2} p \frac{\varepsilon_{\Gamma} m^{2}-\left(p+\frac{k}{2}\right)\left(p-\frac{k}{2}\right)}{\left.\left(\left(p+\frac{k}{2}\right)^{2}+m^{2}\right)\left(p-\frac{k}{2}\right)^{2}+m\right)}
\end{aligned}
$$

where

$$
\begin{aligned}
\varepsilon_{\Gamma} & =1 \text { for } \Gamma=1 \text { and } \varepsilon_{\Gamma}=-1 \text { for } \Gamma=i \gamma_{5} \\
\tilde{\Phi}_{g, k}(k) & =\frac{1}{(2 \pi)^{2}} \int e^{i k x} \Phi_{g, k}(x) d^{2} x .
\end{aligned}
$$

In (A.1) of course the p-integral is logarithmically divergent. We consider instead

$$
\frac{1}{2} \operatorname{Tr}_{\text {reg }} K_{\kappa}^{2}=\int d^{2} k\left|\tilde{\Phi}_{g, \kappa}(k)\right|^{2} F_{\text {reg }}(k),
$$

where (with $\left.p_{ \pm}=p \pm \frac{k}{2}\right)$

Similarly

$$
F_{\text {reg }}(k)=\int d^{2} p\left[\frac{\varepsilon_{\Gamma} m^{2}-p_{+} p_{-}}{\left(p^{+2}+m^{2}\right)\left(p_{-}^{2}+m^{2}\right)}+\frac{1}{\left(p^{2}+m^{2}\right)}\right] .
$$

$$
\frac{1}{2} \operatorname{Tr}_{\mathrm{reg}} K_{\kappa} K_{\kappa}^{*}=\int d^{2} k\left|\tilde{\Phi}_{g, \kappa}(k)\right|^{2} G_{\mathrm{reg}}(k),
$$


where

$$
G_{\mathrm{reg}}(k)=\int d^{2} p\left[\frac{1}{\left(p_{+}{ }^{2}+m^{2}\right)^{\frac{1}{2}}\left(p_{-}{ }^{2}+m^{2}\right)^{\frac{1}{2}}}-\frac{1}{p^{2}+m^{2}}\right] .
$$

Note that not only formally the subtraction term in (A.4) is the negative of the one in (A.6), but actually

$$
\begin{aligned}
\operatorname{Tr}\left(K_{\kappa}{ }^{2}+K_{\kappa} K_{\kappa}^{*}\right) & =\frac{1}{2} \operatorname{Tr}\left(K_{\kappa}+K_{\kappa}^{*}\right)^{2} \\
& =2 \int d^{2} k\left|\tilde{\Phi}_{g, k}(k)\right|^{2}\left(F_{\text {reg }}(k)+G_{\text {reg }}(k)\right)
\end{aligned}
$$

which means that the left hand side of (A.7) is finite without any subtraction. By simple power counting it can be seen that

$$
\frac{1}{2} B_{1}=\frac{1}{k^{2}+\mu^{2}} g\left(F_{\mathrm{reg}}+G_{\mathrm{reg}}\right) g
$$

(here by $g$ we mean the operator of multiplication by $g$ in $x$-space, by $F_{\text {reg }}+G_{\text {reg }}$ multiplication by $F_{\text {reg }}+G_{\text {reg }}$ in momentum space) is a (self-adjoint) HilbertSchmidt operator on $\mathscr{H}_{1}$; therefore

$$
\begin{aligned}
\frac{1}{4} \operatorname{Tr}:\left(K_{\kappa}+K_{\kappa}^{*}\right)^{2}:=:\left(\Phi_{\kappa},\right. & \left.B_{1} \Phi_{\kappa}\right)_{1}: \\
& \rightarrow:\left(\Phi, B_{1} \Phi\right)_{1}: \text { in } L^{2}\left(d \mu_{0}\right) \text { as } \kappa \rightarrow \infty
\end{aligned}
$$

which is (3.18).

In order to show (3.19), we need the explicit form of $F_{\text {reg }}$ and $G_{\mathrm{reg}}$. The integrals (A.4), (A.6) can easily be computed by Bogoliubov's exponential method [17]

$$
\frac{1}{a^{\alpha}}=\frac{1}{\Gamma(\alpha)} \int_{0}^{\infty} d x x^{\alpha-1} e^{-x a}
$$

The results are

$$
\begin{aligned}
& F_{\text {reg }}(k)=2 \pi \sqrt{\frac{\varrho}{4+\varrho}} \ln \frac{\sqrt{\varrho}+\sqrt{4+\varrho}}{2}\left(1+\frac{4}{\varrho}\left(1+\varepsilon_{\Gamma}\right)\right), \\
& G_{\text {reg }}(k)=-\pi \ln \left(1+\frac{\varrho}{4}\right)-2 \pi \ln \frac{1+\sqrt{1-\frac{\varrho}{4+\varrho}}}{2}
\end{aligned}
$$

where

$$
\varrho=\frac{k^{2}}{m^{2}} .
$$

The asymptotic behavior of these expressions is

$$
\begin{aligned}
F_{\text {reg }} & =\pi \ln \varrho+O\left(\frac{1}{\varrho} \ln \varrho\right) \\
G_{\text {reg }} & =-\pi \ln \varrho+4 \pi \ln 2+O\left(\frac{1}{\sqrt{\varrho}}\right), \\
F_{\text {reg }}+G_{\text {reg }} & =4 \pi \ln 2+O\left(\frac{1}{\sqrt{\varrho}}\right) .
\end{aligned}
$$


Now according to (A.7)

$$
\begin{array}{r}
\int \operatorname{Tr}\left(K_{\kappa}+K_{\kappa}^{*}\right)^{2} d \mu_{0}=4 \int d^{2} k\left(F_{\text {reg }}(k)+G_{\text {reg }}(k)\right) \\
\cdot \int d \mu_{0}\left|\tilde{\Phi}_{g, k}(k)\right|^{2} ; \\
\int d \mu_{0}\left|\tilde{\Phi}_{g, \kappa}(k)\right|^{2}=c_{0} \int_{p^{2} \leqq \kappa^{2}} d^{2} p|\tilde{g}(p-k)|^{2} \frac{1}{p^{2}+\mu^{2}} .
\end{array}
$$

(A.15)-(A.17) show that

$$
\begin{aligned}
\int d \mu_{0} \operatorname{Tr}\left(K_{\kappa}+K_{\kappa}^{*}\right)^{2}= & c_{1}+c_{2} \int d^{2} k|\tilde{g}(k)|^{2} \\
& \cdot \int_{p^{2} \leqq \kappa^{2}} d^{2} p \frac{1}{p^{2}+\mu^{2}} \\
= & c_{1}+c_{3} \ln \frac{\kappa^{2}+\mu^{2}}{\mu^{2}} \leqq c_{1}+2 c_{3} \ln \frac{\kappa}{\mu}+c_{3} \frac{\mu^{2}}{\kappa^{2}}
\end{aligned}
$$

from which (3.19) follows. Let us now turn to Eqs. (3.10) and (3.31). According to the definition (2.11)

and therefore

$$
\frac{1}{2} B_{M}=\frac{1}{k^{2}+\mu^{2}} g\left(F_{\text {reg }}+\frac{M^{2}}{2 \lambda^{2}}\right) g
$$

$$
B_{M}-B_{1}=\frac{1}{k^{2}+\mu^{2}} g\left(\lambda^{-2} M^{2}-2 G_{\mathrm{reg}}(k)\right) g
$$

which is Eq. (3.31).

\section{B. Localized Monomials in the Free Bose Field}

Let $F: \mathbb{R}^{2 n} \rightarrow \mathbb{C}$ be a function with the following properties.

a) $F$ has support in $\Lambda \times \cdots \times \Lambda$ ( $n$ times) where $\Lambda$ is a compact subset of $\mathbb{R}^{2}$.

b) $F \in L^{1}\left(\mathbb{R}^{2 n}\right)$.

c) $\sum_{\sigma \in \mathfrak{S}_{2 n}} \int\left|\hat{F}\left(p_{1}, \ldots, p_{n}\right) \tilde{F}\left(p_{n+1}, \ldots, p_{2 n}\right)\right|$

$$
\begin{aligned}
& \cdot \delta\left(p_{\sigma(1)}-p_{\sigma(2)}\right) \ldots \delta\left(p_{\sigma(2 n-1)}-p_{\sigma(2 n)}\right) \\
& \cdot \prod_{k=1}^{n} \frac{1}{p_{\sigma(2 k-1)}^{2}+\mu^{2}} d^{2} p_{1} \ldots d^{2} p_{2 n}<\infty
\end{aligned}
$$

where $\mathfrak{S}_{2 n}$ is the group of permutations of $\{1,2, \ldots, 2 n\}$. Under these conditions we can define a random variable $\mu_{F}$ by

where

$$
u_{F}=L^{2}-\lim _{\kappa \rightarrow \infty} u_{F}^{(\kappa)},
$$

$$
\begin{aligned}
u_{F}^{(\kappa)} & =\int F\left(x_{1}, \ldots, x_{n}\right) \Phi_{\kappa}\left(x_{1}\right) \ldots \Phi_{\kappa}\left(x_{n}\right) d^{2} x_{1} \ldots d^{3} x_{n}, \\
\Phi_{\kappa}(x) & =\left(\Phi * h_{\kappa}\right)(x), \\
h_{\kappa}(x)=\int \quad \int h_{\kappa}(x) & =\frac{1}{(2 \pi)^{2}} \int_{p^{2} \leqq \kappa^{2}} e^{i p x} d^{2} p .
\end{aligned}
$$


Assumption c) just guarantees the existence of the limit in (B.2):

$$
\begin{aligned}
\int\left|u_{F}^{(\kappa)}-u_{F}^{\left(\kappa^{\prime}\right)}\right|^{2} d \mu_{0}= & \int\left|\mu_{F}^{(\kappa)}\right|^{2} d \mu_{0} \\
& +\int\left|u_{F}^{\left(\kappa^{\prime}\right)}\right|^{2} d \mu_{0}-2 \operatorname{Re} \int \overline{u_{F}^{(\kappa)}} u_{F}^{\left(\kappa^{\prime}\right)} d u_{0} \\
& \rightarrow 0 \quad\left(\kappa, \kappa^{\prime} \rightarrow \infty\right)
\end{aligned}
$$

(all integrals over $d \mu_{0}$ can be done and lead to expressions similar to (B.1); the interchange of the limit with the $p$-integration is justified by the dominated convergence theorem).

We call $u_{F}$ a monomial of degree $n$ in the field $\Phi$.

The set $M_{n}$ of monomials of degree $n$ obviously is a linear subspace of $L^{2}\left(d \mu_{0}\right)$ but it is not closed in general. We do not want to close it, since the closure $\overline{M_{n}}$ in general contains functions which are quite different from what one would like to call a monomial of degree $n$.

For example

$$
1 \in \overline{M_{2}} \text {. }
$$

This can be seen as follows:

Let $f_{1}, f_{2}, f_{3}, \ldots$ be an orthonormal sequence in $\mathscr{H}_{-1}$. Then

But

$$
\begin{gathered}
u_{N}=\frac{1}{\ln N} \sum_{n=1}^{N} \frac{1}{u} \Phi\left(f_{n}\right)^{2} \in M_{2} . \\
u_{N} \rightarrow 1 \quad(N \rightarrow \infty) \text { in } L^{2} .
\end{gathered}
$$

Our main result is the following:

Proposition. If $u \in M_{n}, \mu_{0}\{|u|<\varepsilon\}>0$ for all $\varepsilon>0$.

Proof. We start with the following trivial observation: Let

$$
v \in L^{1}\left(d \mu_{0}\right), \quad v \geqq 0, \quad \int v d \mu_{0}=1,
$$

$\int|u|^{2} v d \mu_{0}=\varepsilon^{2}$. Then $\mu_{0}\left\{|u|^{2}<\varepsilon^{2}\right\}>0$.

All we have to do is therefore to find a suitable $v$. Consider the following operator in $\mathscr{H}_{-1}$ :

$$
A_{\kappa, \Lambda}=e_{\Lambda} \theta_{\kappa} e_{\Lambda},
$$

where $e_{\Lambda}$ is the projection onto the subspace of $\mathscr{H}_{-1}$ supported in $\Lambda, \theta_{k}$ is the projection onto the subspace of $\mathscr{H}_{-1}$ which has momentum space support in the ball $p^{2} \leqq \kappa^{2}$.

$A_{\kappa, \Lambda}$ has the following properties:

a)

b)

c)

$$
\begin{gathered}
A_{\kappa, \Lambda} \geqq 0 . \\
1-A_{\kappa, \Lambda}>0 . \\
A_{\kappa, \Lambda} \text { is trace class . }
\end{gathered}
$$

Proof. a) is trivial.

b)

$$
1-A_{\kappa, \Lambda}=\left(1-e_{\Lambda}\right)+e_{\Lambda}\left(1-\theta_{\kappa}\right) e_{\Lambda} \geqq 0 .
$$

Assume $\left(1-A_{\kappa, \Lambda}\right) f=0$ for some $f \in \mathscr{H}_{-1}$. Because of (B.13) this implies $\left(1-e_{\Lambda}\right) \cdot f=0$ and $e_{\Lambda}\left(1-\theta_{\kappa}\right) e_{\Lambda} f=0$. Therefore supp $f \subset A$ and

$$
\left(f,\left(1-\theta_{\kappa}\right) f\right)=0
$$


or

hence

$$
\int_{p^{2} \geqq \kappa^{2}}|\tilde{f}(p)|^{2} \frac{d^{2} p}{p^{2}+\mu^{2}}=0
$$

$$
\tilde{f}(p)=0 \quad \text { for } \quad p^{2} \geqq \kappa^{2} .
$$

But $\tilde{f}$ is an entire function, therefore this implies $\tilde{f}=0$, which completes the proof of $b)$.

c) Let $g \in C_{0}^{\infty} ; g(x)=1$ for $x \in \Lambda$ and let $g$ also denote the operator of multiplication by $g$.

Then

$$
\begin{aligned}
\operatorname{Tr} e_{A} \theta_{\kappa} e_{\Lambda} & =\operatorname{Tr} e_{\Lambda} g^{*} \theta_{\kappa} g e_{\Lambda} \\
& \leqq \operatorname{Tr} g^{*} \theta_{\kappa} g=(2 \pi)^{-4} \int_{q^{2} \leqq \kappa^{2}}|\tilde{g}(p-q)|^{2} \frac{p^{2}+\mu^{2}}{q^{2}+\mu^{2}} d^{2} p d^{2} q
\end{aligned}
$$

[this is true because $e_{\Lambda} g^{*} \theta_{\kappa} g e_{\Lambda}=C^{*} C$ with $C=\theta_{\kappa} g e_{\Lambda}$ and therefore the trace is given by the integral appearing at the end of (B.17)]. $\left(g^{*}\right.$ denotes the adjoint of $g$ as an operator on $\mathscr{H}_{-1}$.)

By using

we obtain

$$
p^{2}+\mu^{2}=q^{2}+\mu^{2}+(p-q)^{2}+2 q \cdot(p-q)
$$

$$
\begin{aligned}
(2 \pi)^{4} \operatorname{Tr} e_{\Lambda} \theta_{\kappa} e_{\Lambda} \leqq & \int_{q^{2} \leqq \kappa^{2}}|\tilde{g}(p-q)|^{2} d^{2} p d^{2} q \\
& +\int_{q^{2} \leqq \kappa^{2}}(p-q)^{2}|\tilde{g}(p-q)|^{2}\left(q^{2}+\mu^{2}\right)^{-1} d^{2} p d^{3} q \\
& +2 \int_{q^{2} \leqq \kappa^{2}}|p-q||q||\tilde{g}(p-q)| 2\left(q^{2}+\mu^{2}\right)^{-1} d^{2} p d^{2} q<\infty
\end{aligned}
$$

since $\tilde{g}$ is strongly decreasing

Because of $b$ ), c)

$$
B_{\kappa, \Lambda}=\left(p^{2}+\mu^{2}\right)^{-1} A_{\kappa, \Lambda}\left(1-A_{\kappa, \Lambda}\right)^{-1}\left(p^{2}+\mu^{2}\right)
$$

is a trace class operator on $\mathscr{H}_{-1}$.

Therefore we can define

$$
\ln \hat{v}_{\kappa, \Lambda}=-\frac{1}{2}\left(\Phi, B_{\kappa, \Lambda} \Phi\right)_{1}
$$

in an obvious way as a $L^{2}$-limit (by approximating $B_{\kappa, \Lambda}$ by finite rank operators).

An elementary calculation shows

$$
\int \hat{v}_{\kappa, \Lambda} d \mu_{0}=\left(\operatorname{det}\left(1-A_{\kappa, \Lambda}\right)\right)^{\frac{1}{2}}
$$

If we set

$$
v_{\kappa, \Lambda}=\left(\operatorname{det}\left(1-A_{\kappa, \Lambda}\right)\right)^{-\frac{1}{2}} \exp \left(-\frac{1}{2}\left(\Phi_{1} B_{\kappa, \Lambda} \Phi\right)_{1}\right)
$$

$v_{\kappa, \Lambda}$ fulfills the conditions (B.9). $v_{\kappa, \Lambda} d \mu_{0}$ is a Gaussian measure with covariance

$$
C_{\kappa, \Lambda}=C_{0}\left(1-e_{\Lambda} \theta_{\kappa} e_{\Lambda}\right) \text {, }
$$

where $C_{0}$ is the free covariance.

If we now compute $\int\left|u_{F}\right|^{2} v_{\kappa, \Lambda} d \mu_{0}$ we can forget about the $e_{\Lambda}$ in (B.24) because of the support properties of $F$. We obtain an expression similar to (B.1), but each 
factor $\frac{1}{p_{j}^{2}+\mu^{2}}$ is replaced by $\frac{1}{p_{j}^{2}+\mu^{2}} \theta\left(p_{j}^{2}-\kappa^{2}\right)$. Because of the finiteness condition (B.1)

$$
\int\left|\mu_{F}\right|^{2} v_{\kappa, \Lambda} d \mu_{0} \rightarrow 0 \quad(\kappa \rightarrow \infty) .
$$

This completes the proof of the proposition.

Lemma 4.2 now follows from the simple observation that $\|K\|_{4}{ }^{4}=\operatorname{Tr}\left(K^{*} K\right)^{2}$ is a localized monomial in the sense defined above.

\section{References}

1. Matthews, P.T., Salam, A.: Nuovo Cimento 12, 563 - 565 (1954); 2, 120-134 (1955)

2. Feynman, R.P.: Rev. Mod. Phys. 20, 367-387 (1948); Phys. Rev. 80, 440-457 (1950)

3. Osterwalder, K., Schrader, R.: Commun. math. Phys. 31, 83-112 (1973); Axioms for Euclidean Green's Functions II (to appear)

4. Osterwalder,K.: In: Velo,G., Wightman,A. (Eds.): Constructive quantum field theory. Lecture notes in physics, Vol. 25, pp. 198-242. Berlin-Heidelberg-New York: Springer 1973

5. Osterwalder, K., Schrader, R.: Helv. Phys. Acta 46, 277-302 (1973)

6. Glimm, J.: Commun. math. Phys. 5, 343-386 (1967); 6, 61-76 (1967)

7. Glimm, J., Jaffe, A.: Ann. Phys. 60, 321-383 (1970); J. Funct. Anal. 7, 323-357 (1971)

8. Schrader, R.: Ann. Phys. 70, 412-457 (1972)

9. Brydges, D., Federbush, P.: A Semi-Euclidean approach to Boson-Fermion model theories. IAS preprint 1973

10. Brydges, D.: Boundedness below for Fermion models, Part 1. University of Michigan preprint, 1974

11. Nelson, E.: J. Funct. Anal. 12, $211-227$ (1973); In: Velo, G., Wightman, A. (Eds.): Constructive quantum field theory. Lecture notes in physics, Vol. 25, pp. 94-124. Berlin-Heidelberg-New York: Springer 1973

12. Guerra, F., Rosen, L., Simon, B.: The $P(\Phi)_{2}$ Euclidean quantum field theory as classical statistical mechanics. Ann. Math. (to appear)

13. Schatten, R.: Norm ideals of completely continuous operators. Ergebnisse der Mathematik und ihrer Grenzgebiete. : 27 (1960)

14. Brascamp, H.J.: Compositio Mathematica 21, 59-80 (1969)

15. Ruston, A.: Proc. London Math. Soc. 53, 109-124 (1951)

16. Bellissard, J.: Private communication

17. Nelson,E.: In Goodman, R., Segal,J. (Eds.): Mathematical theory of elementary particles. MIT Press 1966

18. Kato,T.: Perturbation theory for linear operators. Berlin-Heidelberg-New York: Springer 1966

19. Greub, W.H.: Multilinear algebra. New York: Springer 1967

20. Doob, J.E.: Stochastic processes. New York: John Wiley 1953

21. Glimm, J., Jaffe, A., Spencer, T.: The Wightman axioms and particle structure in the $P(\Phi)_{2}$ quantum field model, Ann. Math. (to appear); In: Velo, G., Wightman,A. (Eds.): Constructive quantum field theory. Lecture notes in physics, Vol. 25, pp. 198-242. Berlin-Heidelberg-New York 1973

22. Dimock, J., Glimm, J.: Measures on the Schwartz distribution space and applications to $P(\Phi)_{2}$ field theories. Adv. Math. (to appear)

23. Bogoliubov, N.N., Shirkov,D.V.: Introduction to the theory of quantized fields. New York: Interscience 1959

24. Schwinger, J. : Phys. Rev. 93, 615 (1953)

Communicated by A.S. Wightman

E. Seiler

Institute for Advanced Study

Princeton, N.J. 08540, USA after July 31 :

Lyman Laboratory for Physics

Harvard University

Cambridge, Mass. 02138, USA 